\title{
Blood pressure measurements in the ankle are not equivalent to blood pressure measurements in the arm
}

\author{
L N Goldstein, ${ }^{1} \mathrm{MB}$ BCh, MMed (Emergency Medicine), FCEM (SA), Cert Critical Care (SA); \\ M Wells, ${ }^{1}$ MB BCh, MSc (Emergency Medicine), Dip PEC (SA), FCEM (SA); K Sliwa, ${ }^{2,3}$ MD, PhD, FESC, FACC, DTM\&H \\ ${ }^{1}$ Division of Emergency Medicine, Faculty of Health Sciences, University of the Witwatersrand, Johannesburg, South Africa \\ ${ }^{2}$ Hatter Institute for Cardiovascular Research in Africa, Department of Medicine, Faculty of Health Sciences, University of Cape Town, \\ South Africa \\ ${ }^{3}$ Soweto Cardiovascular Research Unit (SOCRU), Department of Medicine, Faculty of Health Sciences, University of the Witwatersrand, \\ Johannesburg
}

Corresponding author: L N Goldstein(drg666@gmail.com)

Background. Blood pressure (BP) is often measured on the ankle in the emergency department (ED), but this has never been shown to be an acceptable alternative to measurements performed on the arm.

Objective. To establish whether the differences between arm and ankle non-invasive BP measurements were clinically relevant (i.e. a difference of $\geq 10 \mathrm{mmHg}$ ).

Methods. This was a prospective cross-sectional study in an urban ED making use of a convenience sample of 201 patients (18 - 50 years of age) who were not in need of emergency medical treatment. BP was measured in the supine position on both arms and ankles with the correct size cuff according to the manufacturer's guidelines. The arm and ankle BP measurements were compared.

Results. There was a clinically and statistically significant difference between arm and ankle systolic BP (SBP) and mean arterial pressure (MAP) (-13 mmHg, 95\% confidence interval (CI) -28 - $1 \mathrm{mmHg}$ and $-5 \mathrm{mmHg}$, 95\% CI - 13 - $4 \mathrm{mmHg}$, respectively), with less difference in diastolic BP (DBP) (2 mmHg, 95\% CI -7 - $10 \mathrm{mmHg}$ ). Only 37\% of SBP measurements and 83\% of MAP measurements were within an error range of $10 \mathrm{mmHg}$, while $95 \%$ of DBP measurements agreed within $10 \mathrm{mmHg}$. While the average differences (or the bias) were generally not large, large variations in individual patients (indicating poor precision) made the prediction of arm BP from ankle measurements unreliable. Conclusion. Ankle BP cannot be used as a substitute for arm BP in the ED.

S Afr Med J 2014;104(12):869-873. DOI:10.7196/SAMJ.8102 


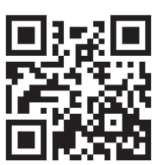

Blood pressure (BP) is an important vital sign in patients admitted to the emergency department (ED) and is used to guide resuscitation of the critically ill or injured patient. Ideally, BP should be measured directly or invasively when precise or continuous monitoring is required, but this can be time consuming and impractical in the resuscitation setting. The utility of non-invasive BP (NIBP) measurements, along with other clinical signs, includes detection of an abnormally high or low BP in symptomatic or asymptomatic patients.

In patients who are not severely ill or injured, it is reasonable to expect that BP measurements will be performed on one of the patient's arms according to standard practice guidelines. ${ }^{[1]}$ This is not necessarily the case with the critically ill or injured patient, who may not be able to be positioned correctly or where access to an arm may not be feasible because of injuries or the presence of intravenous catheters. Healthcare providers sometimes then place the BP cuff on the patient's ankle. There is limited evidence regarding the interchangeable use of arm and ankle BPs in the ED, or in other clinical settings. Similarly, the NIBP machines that are generally used in clinical settings are unlikely to be validated for use on the ankle.

As early as 1925, it was found that the systolic BP was 20 - 40 $\mathrm{mmHg}$ higher in the leg than in the arm in normal subjects at rest. ${ }^{[2]}$ This is the premise on which the ankle-brachial index (ABI) is employed to non-invasively determine whether patients have peripheral arterial disease. Normal values for this ratio are considered to be between 0.9 and 1.3. It is abnormal if the ABI is $\leq 0.9$. The prevalence of peripheral arterial disease increases with age. The prevalence in patients older than 55 years is $\sim 16 \%$, whereas that in patients older than 85 years is $\sim 22 \% .{ }^{[3]}$ By applying the normal ABI ratio to an arm systolic BP (SBP) of $120 \mathrm{mmHg}$, the measured ankle SBP would be considered normal if it is anywhere between 108 and $156 \mathrm{mmHg}$, i.e. up to a $36 \mathrm{mmHg}$ difference, which is certainly clinically significant.

In a study looking at the differences in BP between the arm and the calf (i.e. proximal leg, not ankle) by Zahn et al., ${ }^{[4]}$ pregnant patients had significant differences in their SBP and diastolic BP (DBP), but no differences in their mean arterial pressure (MAP). There was, however, a large degree of variability among the patients and a tendency for the SBP to be higher in the arm than in the calf and the DBP to be lower in the calf than in the arm. Sanghera et al. ${ }^{[5]}$ found no association between arm and ankle BPs in pregnant patients during caesarean section. Although the average differences for SBP, MAP and DBP that they observed were below clinical significance, the limits of agreement were wide. This would have resulted in hypotension being missed in $20 \%$ of the patient population, with potentially dire consequences. This finding confirmed that the ankle position is not an alternative option in pregnant patients. ${ }^{[5]}$

Anaesthetised children have also been found to have inconsistent results with arm and ankle BP measurements. In children 8 years and younger, BPs were found to be lower in the leg than in the arm, ${ }^{[6]}$ whereas another study showed no link between arm and ankle BP. ${ }^{[7]}$

Wilkes and DiPalma ${ }^{[8]}$ advised that although ankle SBPs and MAPs were higher than those in the arm in their cohort of anaesthetised patients, the ankle was an acceptable alternative should the arm not be available. Conversely, Block and Schulte ${ }^{[9]}$ found that although the SBP was higher in the ankle than in the arm, the MAPs were statistically equivalent, suggesting that ankle cuff placement is a reliable alternative. The inconsistency in the results of these studies does not support the routine use of ankle BP measurements in management decisions for critically and injured patients.

BP measurements are fundamental to management of patients in the resuscitation setting in the ED. Whether there is a clinically significant difference between arm and ankle BPs in this setting has not yet been determined. Before assessing this in the hypo- or hypertensive seriously ill or injured patient, the equivalence should be evaluated in haemodynamically normal patients.

\section{Methods}

This was a prospective cross-sectional study of a convenience sample of adult patients presenting to an urban ED from 21 October 2010 to 19 January 2011. Patients with significant cardiovascular pathology or musculoskeletal abnormalities, pregnant women, and patients known to have peripheral arterial disease or diabetes were excluded. The data collection process is shown in Fig. 1.

The research was approved by the Human Research Ethics Committee of the Faculty of Health Sciences, University of the Witwatersrand. Written informed consent was obtained from the patients before enrolment.

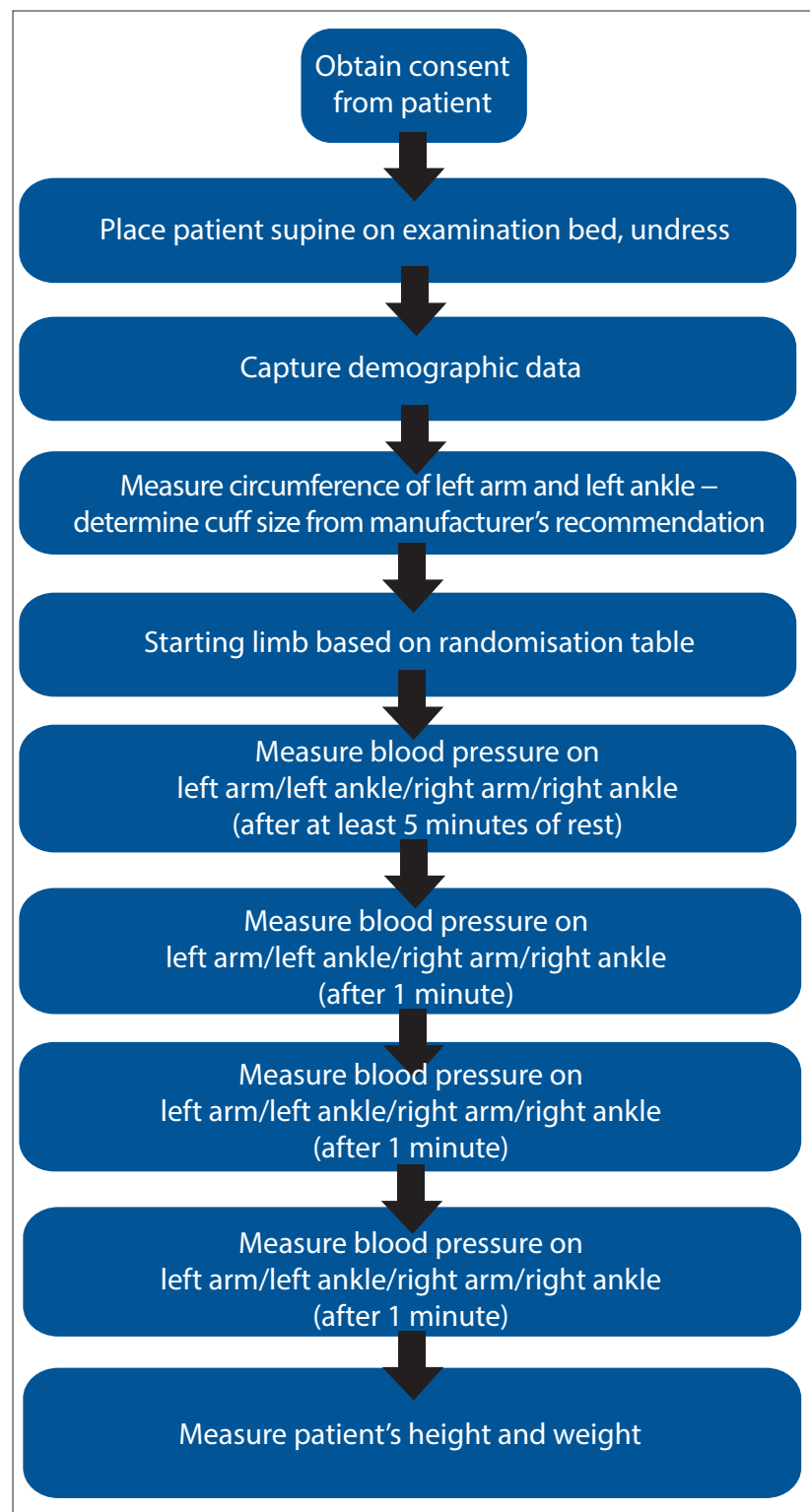

Fig. 1. Procedure used for data collection. 
Table 1. Basic demographic data

\begin{tabular}{llll}
\hline & $\begin{array}{l}\text { Total } \\
(\boldsymbol{N = 2 0 1})\end{array}$ & $\begin{array}{l}\text { Males } \\
(\boldsymbol{n}=\mathbf{1 3 8})\end{array}$ & $\begin{array}{l}\text { Females } \\
(\boldsymbol{n}=\mathbf{6 3})\end{array}$ \\
\hline Age (years), mean $(95 \% \mathrm{CI})$ & $34(21-49)$ & $34(21-49)$ & $35(22-49)$ \\
Height $(\mathrm{cm})$, mean $(95 \% \mathrm{CI})$ & $171(154-186)$ & $175(164-188)$ & $161(150-174)$ \\
Weight $(\mathrm{kg})$, mean $(95 \% \mathrm{CI})$ & $74(52-109)$ & $75(53-100)$ & $72(46-111)$ \\
BMI $\left(\mathrm{kg} / \mathrm{m}^{2}\right)$, mean $(95 \% \mathrm{CI})$ & $25.6(18.1-36.2)$ & $24.6(18.2-33.1)$ & $27.8(17.9-41.5)$ \\
Arm circumference $(\mathrm{cm})$, mean $(95 \% \mathrm{CI})$ & $29(23-36)$ & $29(23-36)$ & $29(23-40)$ \\
Ankle circumference $(\mathrm{cm})$, mean $(95 \% \mathrm{CI})$ & $27(22-34)$ & $27(22-32)$ & $28(23-35)$ \\
Trauma, $n(\%)$ & $136(67.7)$ & $109(79.0)$ & $27(42.9)$ \\
Non-trauma, $n(\%)$ & $65(32.3)$ & $29(21.0)$ & $36(57.1)$ \\
CI $=$ confidence interval; BMI = body mass index. & & &
\end{tabular}

\section{Measuring instrument}

The NIBP was measured with a GE Healthcare Carescape V100 Vital Signs Monitor. SBP, DBP, MAP and the heart rate were recorded. This is a typical NIBP device used in the ED that might be used on the ankle in the clinical setting, despite not being validated for this purpose. The machine was reset after each reading.

\section{Data analysis}

In line with previous studies, this study made use of Bland-Altman, ${ }^{[10]}$ actual and absolute (root mean square) differences and categorical analysis. The paired $t$-test was used for comparisons of paired parametric data and the McNemar test for paired categorical data. The $t$-test was used for unpaired parametric data and Fisher's exact test for unpaired categorical data.

\section{Results}

Two hundred and one adult patients were enrolled into the study. There were 138 males (68.7\%) and 63 females (31.3\%). The basic demographic data are summarised in Table 1.

There were statistically significant differences in BP measurements between the male and female patients, although further subgroup analysis showed no change in the relationship between arm and ankle BPs. There were no statistically significant differences between the left and right sides for either the arm or the ankle position. The order in which the BPs were measured did not affect the comparison between the arm and the ankle readings.

In almost $91 \%$ of the study population, the same cuff could be used for both the patient's arm and ankle. One-third of fatter patients and one-third of thinner patients had an arm cuff size that differed from their ankle cuff size. This meant that different cuffs were required for the BP measurements on their arms.

There was a statistically and clinically significant difference between arm and ankle SBP in both males and females. There was a statistically but not clinically significant difference between arm and ankle DBP and MAP in males and females (Table 2).

The mean actual, absolute (root mean square) and percentage differences between the arm and ankle SBPs were clinically significantly different, while those between the arm and ankle DBPs were within the clinically acceptable range. The mean actual, absolute and percentage differences between the arm and ankle MAPs were within the clinically acceptable range, but the range of the confidence interval (CI) was not (Table 3, and Fig. 2 for the Bland-Altman graphic representation).

Non-parametric analysis of differences between the arm and the ankle BPs by category showed that $40 \%$ of SBP, $5 \%$ of DBP and $16 \%$ of MAP measurements differed by $>10 \mathrm{mmHg}$ (Fig. 3).
Table 2. Differences between mean arm and mean ankle BPs*

\begin{tabular}{|c|c|c|c|}
\hline & \multicolumn{2}{|c|}{ BP (mmHg) } & \multirow[b]{2}{*}{$p$-value ${ }^{\dagger}$} \\
\hline & Arm & Ankle & \\
\hline \multicolumn{4}{|l|}{ SBP } \\
\hline Male & 126 & 141 & $<0.0001$ \\
\hline Female & 117 & 127 & $<0.0001$ \\
\hline All & 123 & 137 & $<0.0001$ \\
\hline \multicolumn{4}{|l|}{ DBP } \\
\hline Male & 74 & 73 & 0.0115 \\
\hline Female & 70 & 67 & $<0.0001$ \\
\hline All & 73 & 71 & $<0.0001$ \\
\hline \multicolumn{4}{|l|}{ MAP } \\
\hline Male & 94 & 100 & $<0.0001$ \\
\hline Female & 88 & 90 & $<0.0001$ \\
\hline All & 92 & 97 & $<0.0001$ \\
\hline \multicolumn{4}{|c|}{$\begin{array}{l}\mathrm{BP}=\text { blood pressure; } \mathrm{SBP}=\text { systolic blood pressure; } \mathrm{DBP}=\text { diastolic blood pressure; } \mathrm{MAP}= \\
\text { mean arterial pressure. } \\
\text { *The differences between blood pressure readings in males and females were also } \\
\text { significantly different for each measurement. } \\
\text { 'Paired } t \text {-test. }\end{array}$} \\
\hline
\end{tabular}

\section{Discussion}

The fact that the ankle BP is generally higher than arm BP has been known for a long time ${ }^{[2]}$ but whether there is a clinically useful and predictable link between the two readings in the haemodynamically normal patient, let alone in the resuscitation setting, has not been evaluated.

In this study, there was a larger difference between arm and ankle SBP in males than in females, with the BPs for both genders being outside the clinically acceptable range. There was no clinically significant difference between the patient age groups or between race groups. The smaller difference in females may be related to their shorter heights and hence a shorter distance between the upper and lower limb arteries.

The mean SBP measured in the ankle was $13 \mathrm{mmHg}$ higher than that measured in the arm. This in itself is not clinically acceptable, but what is more worrying is that this difference is not constant between patients. The ankle SBP was up to $28 \mathrm{mmHg}$ higher, or conversely $1 \mathrm{mmHg}$ lower, than the measured SBP in the arm (95\% CI). This difference in SBP translates to a mean percentage difference of $11 \%$. These large differences are similar to those predicted by a normal 

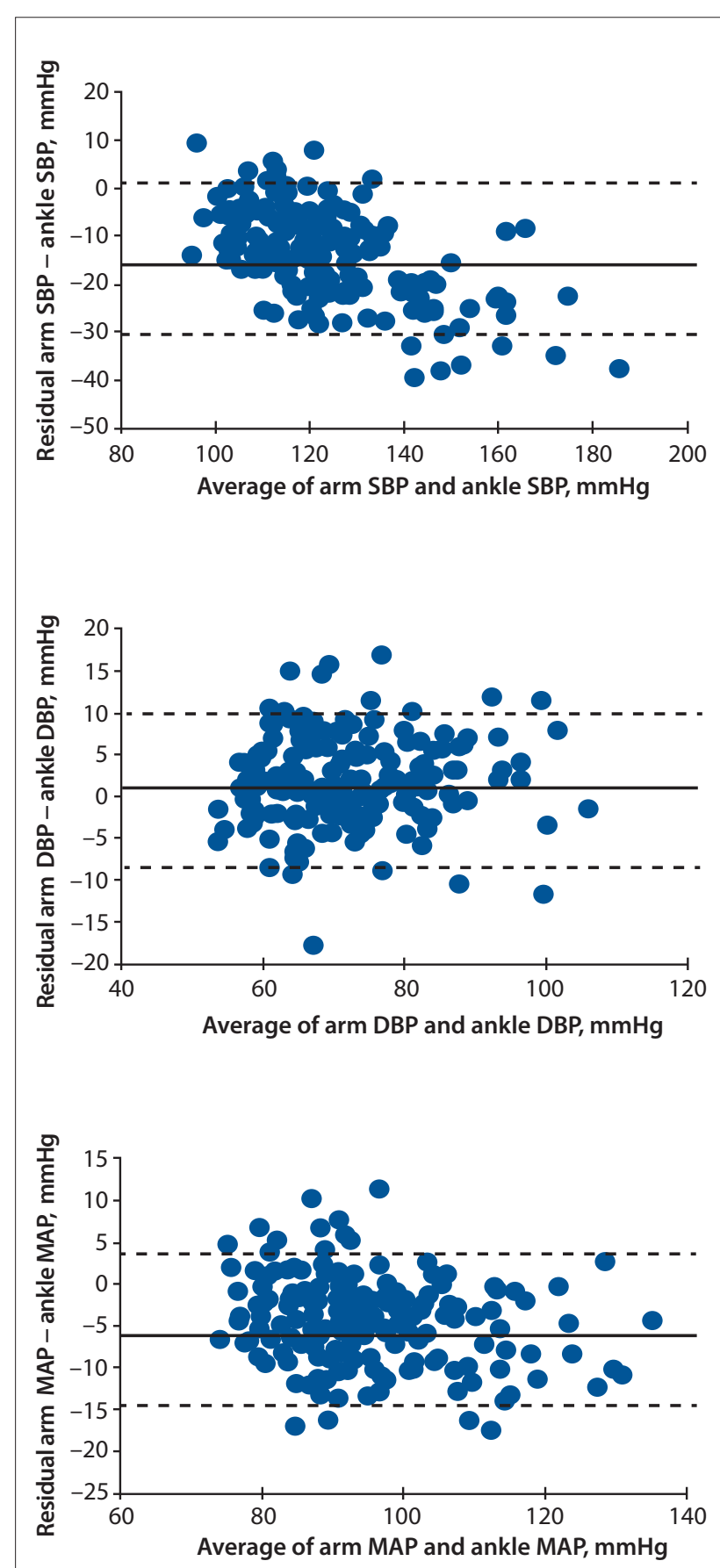

Fig. 2. Bland-Altman plots of arm v. ankle systolic, diastolic and mean BPs $(B P=$ blood pressure; $S B P=$ systolic blood pressure; $D B P=$ diastolic blood pressure; $M A P=$ mean arterial pressure.)
ABI. ${ }^{[3]}$ Ankle SBP therefore cannot be used to reliably estimate the SBP in the individual.

Surprisingly, DBP fared best, with the absolute and actual differences between the arm and the ankle being $4 \mathrm{mmHg}$ and 2 $\mathrm{mmHg}$, respectively. The ankle reading was on average only $2 \%$ higher than the measured arm DBP. This is surprising, because the oscillatory BP machine measures the MAP and calculates the SBP and DBP. The CI for the absolute average DBP extended beyond the clinically significant range of $10 \mathrm{mmHg}$, however, with the actual $\mathrm{CI}$ range being $-7-10 \mathrm{mmHg}$. This means that a measured DBP at the ankle could be $7 \mathrm{mmHg}$ lower, or up to $10 \mathrm{mmHg}$ higher, than that in the arm. While within the 'clinically acceptable' range, the fact that the measured DBP can be higher or lower than the true arm DBP is potentially problematic should decisions be based on that measured DBP. However, DBP is rarely the value on which clinical decisions are based in the emergency setting.

The MAP was also statistically significantly different between the arm and the ankle, with higher MAPs noted in the ankle. The MAP actual mean difference between the arm and the ankle fell within the clinically acceptable error range of $10 \mathrm{mmHg}$, but was $5 \mathrm{mmHg}$ higher than the mean arm MAP. Unfortunately, the 95\% CIs show that the range extends beyond the acceptable range, with values up to $13 \mathrm{mmHg}$ higher in the ankle than in the arm. The percentage difference in the ankle was on average $5 \%$, but up to $20 \%$ higher or $6 \%$ lower than true arm values.

Sixty per cent of SBP measurements in the ankle were $>10 \mathrm{mmHg}$ different from the arm SBP. Conversely, DBP ankle readings were within $10 \mathrm{mmHg}$ of the arm DBP in the majority of cases (95\%). MAP ankle readings were also within $10 \mathrm{mmHg}$ of the arm MAP in the majority of cases (84\%). Arm-ankle differences in SBP tended to be higher if the measured SBP was $\leq 150 \mathrm{mmHg}$, but were greater than the clinically acceptable error of $10 \mathrm{mmHg}$. Where the SBP exceeded $150 \mathrm{mmHg}$, the measured ankle SBP was always higher than the arm, but was an alarming $24 \mathrm{mmHg}$ (on average) to $38 \mathrm{mmHg}$ different. The higher the BP, the more inaccurate the SBP became. This was reaffirmed in the MAP differences. DBP was more reliable across the SBP range, but performed best at SBP $\leq 120 \mathrm{mmHg}$. These findings may be beneficial with regard to DBP measurement in hypotensive patients, but since the lowest recorded SBP was $92 \mathrm{mmHg}$ in this study, accuracy of the DBP requires validation in a more severely hypotensive population.

There are various explanations for the generally higher ankle SBP than arm SBP: (i) following the law of LaPlace, BP is increased as a result of the higher resistance from the decreased radius of more distal vessels; (ii) arterial pulsations may be dampened distally; (iii) ankle readings may be inaccurate owing to inability of the cuff to compress the dorsalis pedis/posterior tibial artery; and (iv) differences in subcutaneous fat and/or muscle may interfere with compression of the artery and detection of the oscillations.

In the emergency setting, the SBP or the MAP is the preferred target. For example, in a hypotensive polytrauma patient, current practice is

Table 3. Mean actual, absolute and percentage differences between arm and ankle BPs

\begin{tabular}{llll}
\hline & & \multicolumn{1}{c}{ Difference, mean $(\mathbf{C I})$} \\
\cline { 2 - 4 } & Arm and ankle SBP (mmHg) & Arm and ankle DBP (mmHg) & Arm and ankle MAP (mmHg) \\
\hline Actual & $-13(-28-1)$ & $2(-7-10)$ & $-5(-13-4)$ \\
Absolute & $14(2-28)$ & $4(1-11)$ & $6(1-13)$ \\
$\%$ & $-11(-22-1)$ & $2(-6-8)$ & $-5(-19-6)$ \\
BP = blood pressure; $\mathrm{CI}=$ confidence interval; SBP = systolic blood pressure; DBP = diastolic blood pressure; MAP = mean arterial pressure.
\end{tabular}




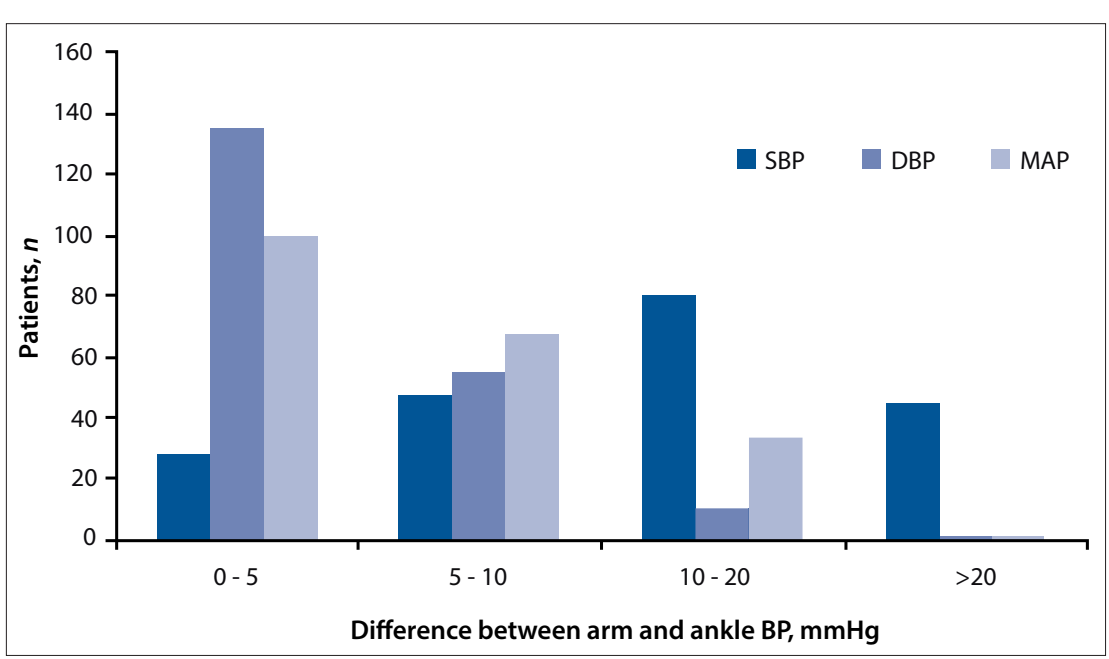

Fig. 3. Error categories of absolute differences in SBP, DBP and MAP between arm and ankle measurements. $(S B P=$ systolic blood pressure; $D B P=$ diastolic blood pressure; $M A P=$ mean arterial pressure; $B P=$ blood pressure.)

not to raise the SBP $>100 \mathrm{mmHg}$ - so-called 'permissive hypotension'. This subsequently evolved to a target MAP of $\sim 65 \mathrm{mmHg},{ }^{[11,12]}$ or even a MAP as low as $50 \mathrm{mmHg}$, as part of 'damage control resuscitation. ${ }^{\text {'13,14] }}$ This is different from a patient with acute coronary syndrome and a hypertensive emergency requiring that the SBP brought down to $<180$ $\mathrm{mmHg}$ to decrease the risk of intracerebral haemorrhage during thrombolysis. The septic patient requires early goal-directed therapy maintenance of the MAP at $65 \mathrm{mmHg}$ with fluids and vasoactive agents as part of shock treatment. ${ }^{[15]}$ In each of these examples, differences between a measured arm and ankle $\mathrm{BP}$ of $>10 \mathrm{mmHg}$ higher or lower would significantly change management goals for each patient and potentially have deleterious effects.

Wilkes and DiPalma ${ }^{[8]}$ suggested that the ankle can be used as an alternative to the arm should the arm not be available, 'recognising that the readings are generally higher than the corresponding brachial pressures'. The problem with their suggestion, as can be seen from our data, is that the 'general rule' does not always apply. A practice suggested by one of the authors of the study by Moore et al. ${ }^{[16]}$ is to initially take the BP in the arm before proceeding to the ankle in order to get an idea of the degree of difference between the two sites - a so-called 'calibration of the ankle BP'. While this may seem a logical option, there are two inherent problems: in the ED it is not always feasible to take the initial arm $\mathrm{BP}$ measurement for comparison, and there is no guarantee that the initial difference in readings noted will remain consistent throughout a range of BPs. The suggestion is that $\mathrm{BP}$ will come to mirror central venous pressure as an indicator of fluid status, and be used as a trend over several readings rather than taken as an absolute, based on only one reading.

\section{Study limitations}

Only patients with non-life-threatening conditions in the ED were evaluated, which may preclude extrapolation of our findings to hypotensive or hypertensive patients in other settings. The BP in the ankle was compared with the NIBP of the arm and not the intra-arterial actual $\mathrm{BP}$, this being the typical scenario in practice.

\section{Conclusions}

A comparison of NIBP in the arm and ankle in patients in the ED has shown that, in general, the ankle NIBP cannot be used interchangeably with the arm NIBP.

The most reliable reading that can be obtained at the ankle as opposed to the arm is the DBP, which unfortunately does not have many clinical applications as a target in the resuscitation setting. Although the ankle can be seen as a convenient alternative site to the arm in the resuscitation environment, there is too much interpatient variability to give a meaningful value.

The search for an accurate, instantaneous, easily repeatable, non-invasive measure of $\mathrm{BP}$ must continue, or an alternative to $\mathrm{BP}$ monitoring be found.

\section{Recommendations}

- Ensure that the correct cuff size is used for BP measurements.

- If the BP cuff was initially placed on the ankle in the resuscitation, ensure that it is transferred to an arm when feasible.

- If the BP cuff is to remain on the ankle, note that the only reliable reading is the DBP.

- Ankle BP readings are more inaccurate with higher BPs.

Funding. No external funding was obtained from any source.

\section{References}

1. Pickering TG, Hall JE, Appel LJ, et al. Recommendations for blood pressure measurement in humans and experimental animals. Part 1: Blood pressure measurement in humans: A statement for professionals from the Subcommittee of Professional and Public Education of the American Heart Association Council on High Blood Pressure Research. Hypertension 2005;45(1):142-161. [http://dx.doi.org/10.1161/01.HYP.0000150859.47929.8e]

2. Burdick W, Clarke N, Garlichs R, et al. Differences in blood pressure in the arm and leg in normal subjects. Am J Physiol 1925;72(1):169-176.

3. Newman AB, Siscovick DS, Manolio TA, et al. Ankle-arm index as a marker of atherosclerosis in the Cardiovascular Health Study. Cardiovascular Heart Study (CHS) Collaborative Research Group. Circulation 1993;88(3):837-845. [http://dx.doi. org/10.1161/01.CIR.88.3.837]

4. Zahn J, Bernstein H, Hossain S, et al. Comparison of noninvasive blood pressure measurements on the arm and calf during cesarean delivery. J Clin Monit Comput 2000;16(8):557562. [http://dx.doi.org/10.1023/A:1012267312308]

5. Sanghera S, North A, Abernethy S, et al. Arm and ankle blood pressure during caesarean section. Int J Obstet Anesth 2006;15(1):24-27. [http://dx.doi.org/10.1016/j.ijoa.2005.04.016]

6. Short JA. Noninvasive blood pressure measurement in the upper and lower limbs of anaesthetized children. Paediatr Anaesth 2000;10(6):591-593. [http://dx.doi.org/10.1111/j.14609592.2000.00558.x]

7. Crapanzano MS, Strong WB, Newman IR, et al. Calf blood pressure: Clinical implications and correlations with arm blood pressure in infants and young children. Pediatrics 1996;97(2):220-224.

8. Wilkes JM, DiPalma JA. Brachial blood pressure monitoring versus ankle monitoring during colonoscopy. South Med J 2004:97(10):939941. [http://dx.doi.org/10.1097/01.SMJ.0000129929.28792.77]

9. Block FE, Schulte GT. Ankle blood pressure measurement, an acceptable alternative to arm measurements. Int J Clin Monit Comput 1996;13(3):167-171. [http://dx.doi. org/10.1023/A:1016997232542]

10. Bland JM, Altman DG. Statistical methods for assessing agreement between two methods of clinical measurement. Lancet 1986:1 (8476) 307 310. [http://dx.doi.org/10.1016/S0140-6736(86)90837-8]

11. Dutton RP, Mackenzie CF, Scalea TM. Hypotensive resuscitation during active hemorrhage: Impact on in-hospital resuscitation during active hemorrhage: Impact on in-hospital
mortality. J Trauma 2002;52(6):1141-1146. [http://dx.doi. mortality. J Trauma 2002;52(6):1141-1146. [http://dx.doi.

2. Bilkovski RN, Rivers EP, Horst HM. Targeted resuscitation strategies after injury. Curr Opin Crit Care 2004;10(6):529-538.

13. Cotton BA, Reddy N, Hatch QM, et al. Damage control resuscitation is associated with a reduction in resuscitation volumes and improvement in survival in 390 damage control derotony patients. Ann Surg 2011,254(4)

14. Morrison CA, Carrick MM, Norman MA, et al. Hypotensive Morrison CA, Carrick MM, Norman MA, et al. Hypotensive
resuscitation strategy reduces transfusion requirements and severe postoperative coagulopathy in trauma patients with conoris controlled trial. J Trauma $2011,70(3) \cdot 6$

5. Rivers E, Nguyen B, Havstad S, et al. Early goal-directed therapy in the treatment of severe sepsis and septic shock. N Engl J Med 2001;345(19):1368-1377. [ittp://dx.doi.org/10.1056/NE)Moa010307] 6. Moore C, Dobson A, Kinagi M, et al. Comparison of blood pressure measured at the arm, ankle and calf. Anaesthesia 2008;63(12):1327-
1331. [http://dx.doi.org/10.1111/j.1365-2044.2008.05633.x]

Accepted 25 July 2014 